\title{
Hypothalamic Neurons Preferentially Respond to Female Nest Coo Stimulation: Demonstration of Direct Acoustic Stimulation of Luteinizing Hormone Release
}

\author{
Mei-Fang Cheng, ${ }^{1}$ Jing Pian Peng, ${ }^{1}$ and Patricia Johnson ${ }^{2}$ \\ ${ }^{1}$ Department of Psychology, Biopsychology Program, Rutgers University, Newark, New Jersey 07102, and \\ 2Department of Animal Science, Cornell University, Ithaca, New York 14853
}

Avian vocalizations are generally understood to play a pivotal role in reproductive functions. The role of the hypothalamus in gonadotropin release in higher vertebrates including birds is well established. To date, however, a direct linkage between the neuronal processing of vocal input and the contingent luteinizing hormone (LH) response has not been demonstrated. In this study, using female ring doves, we recorded neuronal activity from hypothalamic nuclei that, as we have shown previously, receive acoustic inputs from the auditory thalamic relay. Concurrently with recording single-unit responses to stimulation with species-specific coo vocalizations, we sampled LH levels in blood from the pituitary veins. LH concentration in the plasma was significantly elevated in birds hearing speciestypical coos but not in birds exposed to experimentally altered coos or white noise or in birds that received no vocal stimulation. We found two types of neurons in the preoptic and anterior hypothalamus that selectively responded to the female nest coo: excitatory units and inhibitory units. Among the excitatory neurons are units characterized by two bursts separated by a period of slow spiking or complete silence, in a pattern approximately corresponding temporally to the two-note coo. We designate them as female-nest-coo-specific units. Most neurons in the posterior hypothalamus were nonselective in their response. Female nest coo and male nest coo stimulation evoked an equal magnitude of discharge changes from responsive units in the preoptic-anterior hypothalamic area. We found, however, that the LH increment was three times greater for birds hearing female nest coos than for birds hearing male nest coos. These observations suggest that feature-detecting neurons such as the female-nest-coo-specific units are involved in gonadotropin-releasing hormone output. The present findings are consistent with the well established behavioral evidence that female nest coos mediate ovarian growth.

Key words: vocalization; sound stimulation of endocrine response; hypothalamus; auditory processing; $\mathrm{GnRH}$; callspecific neuronal discharge
Neuroendocrine control of reproduction is remarkably conserved throughout the vertebrate subphylum (Muske, 1993). Of the various neuropeptides and neurotransmitters synthesized in the hypothalamus that are involved in reproduction, the gonadotropinreleasing hormone $(\mathrm{GnRH})$ controls the release of luteinizing hormone $(\mathrm{LH})$ from the anterior pituitary and triggers the cascade of endocrine responses that culminate in ovulation (Silverman et al., 1994). This exquisite machinery is adapted to the particular environmental niche and social organization of each species (Marshall, 1942; Lehrman, 1961; Bronson, 1989; Ball, 1993; Wingfield et al., 1994). Successful breeding thus relies on selective processing and encoding of relevant sensory information within the hypothalamus. The importance of communication signals in reproduction has been shown in a wide variety of vertebrate classes (Wingfield et al., 1994). Vocal communication is one of the forms most intensively studied, especially in frogs and birds (Capranica and Moffatt, 1983; Cheng, 1992, 1993; Wilczynski et al., 1994). In many bird species, courtship calls play

\footnotetext{
Received Oct. 10, 1997; revised April 17, 1998; accepted April 23, 1998.

This work was supported by NIMH Grant MH-47010 to M.F.C. We thank Carrie Brooks for excellent technical assistance. We are grateful to Dr. Peter Sharp (Agriculture and Food Research Council Poultry Research Centre) for the reagents for the chicken LH RIA. We also thank Dr. Marylou Glasier for critical reading of an earlier version of this manuscript and Dr. Michael Casey for helpful discussion of experimental results.

Correspondence should be addressed to Dr. Mei-Fang Cheng, Rutgers University, Department of Psychology, 101 Warren Street, Newark, NJ 07102.

Copyright (C) 1998 Society for Neuroscience $\quad 0270-6474 / 98 / 185477-13 \$ 05.00 / 0$
}

a crucial role in breeding success (Lehrman, 1961; Kroodsma, 1976; Wingfield et al., 1994). In the ring dove (Streptopelia risoria), the male typically initiates courtship with bow cooing and nest cooing, to which the female responds with her own nest cooing. In 5-7 d after the female begins nest cooing, she lays a clutch of one or two eggs. Muting the female blocks her own ovarian growth, whereas playing back the female's nest coo reinstates such growth (Cheng, 1992), and a deafened female's movements associated with the nest-coo display can stimulate moderate ovarian growth (Cheng et al., 1988). These findings suggest that both auditory cues and the motor act of displaying are involved in ovarian development.

To determine the neuronal networks that subserve these endocrine effects of nest coos, we applied anterograde and retrograde tracing methods. We found massive hypothalamic afferent projections from the shell region of the auditory thalamic nucleus (Durand et al., 1992) and minor projections from the midbrain vocal control nucleus (Cheng and Zuo, 1994). That the hypothalamic recipients of these projections respond to sound was subsequently confirmed in an antidromic electrophysiological study (Cheng and Peng, 1997). These findings provide the impetus for the present test of the hypothesis that coo-sensitive neuronal units in the hypothalamus are involved in $\mathrm{LH}$ release. We demonstrate biologically significant sound stimulation of LH release by combining playbacks of the species-specific courtship call, recording of hypothalamic neuronal activity, and sampling of anterior pituitary secretory output in the same experimental preparation. 


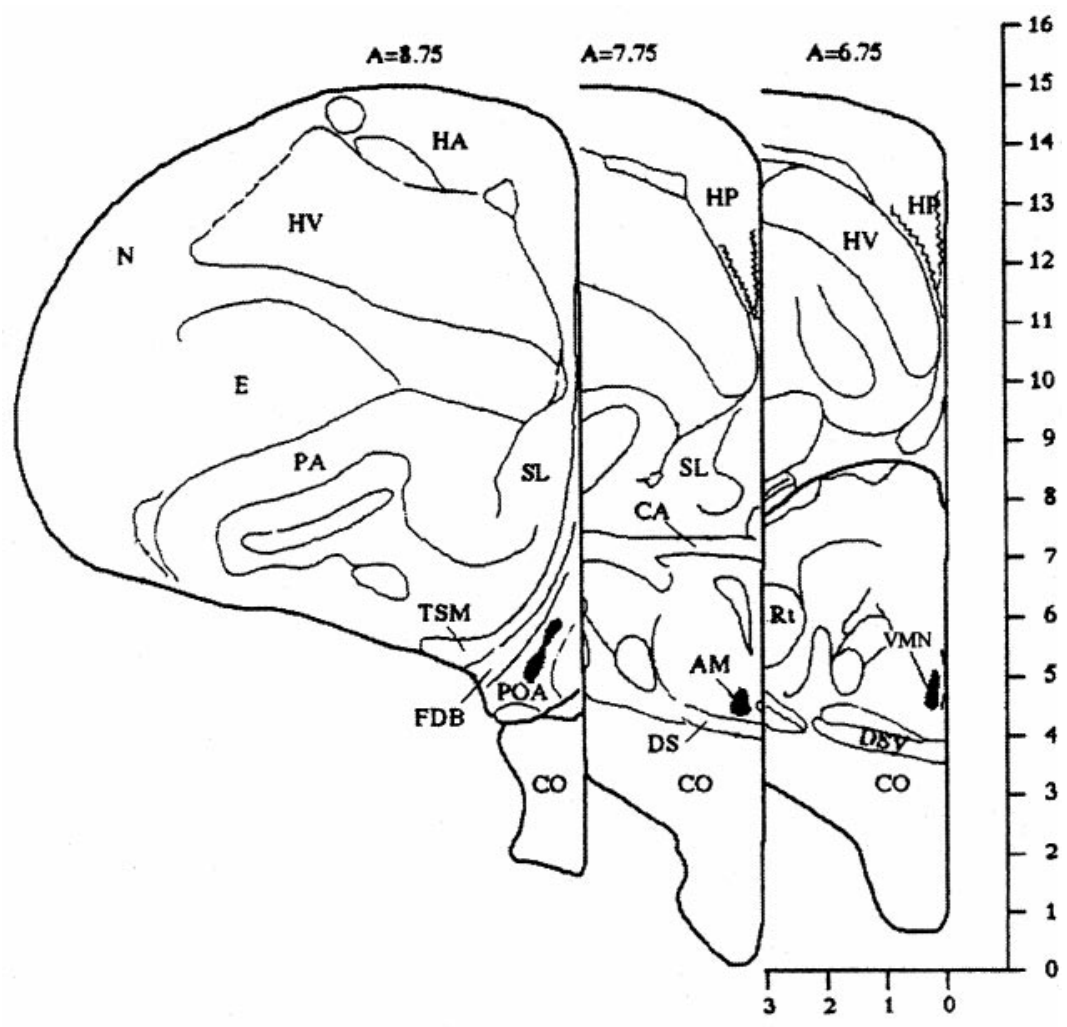

Figure 1. Position of recording sites in the hypothalamus. Top panel, Distribution of recording sites (shaded areas) in the preoptic area $(P O A)$, the anterior medial hypothalamus $(A M)$, and the ventromedial nucleus ( $V M N$, also known as the posterior medial hypothalamus). Bottom panel, Photomicrograph of the histological location of one recording electrode (arrow) in the AM area.

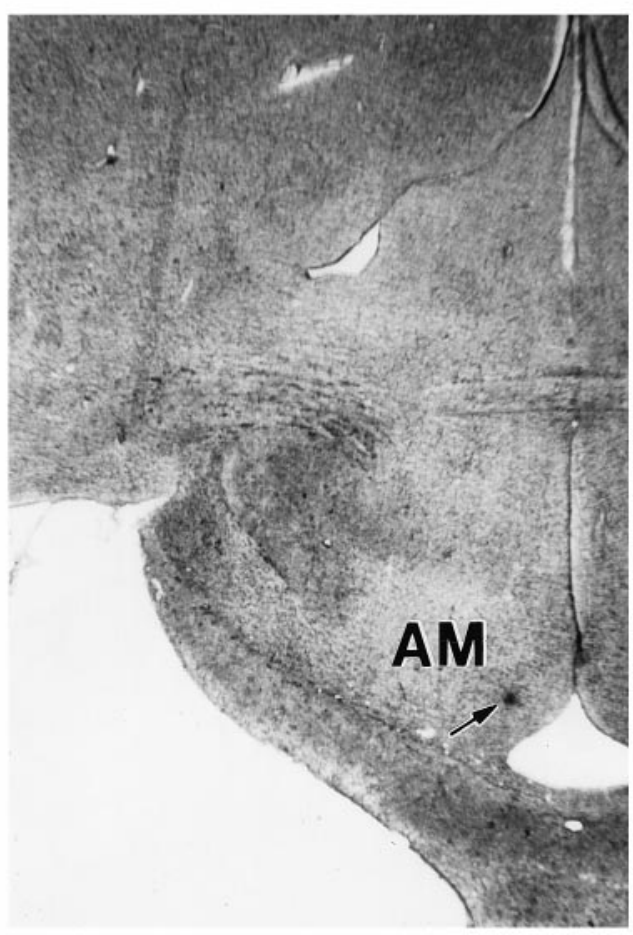

\section{MATERIALS AND METHODS}

Subjects. Ring doves (Streptopelia risoria) were bred at the Institute of Animal Behavior facility at Rutgers University under a 14/10 hr light/ dark photoperiod. At the time of testing, the birds were 135-150 d of age and weighed 150-180 gm. A different set of birds was used in each of two experiments: 23 females and 1 male were used in experiment I, and 11 females and 2 males were used in experiment II. All birds in the experiments were anesthetized with urethane $(20 \% \mathrm{w} / \mathrm{v}$ solution, 0.4

$\mathrm{ml} / \mathrm{kg}$, i.m.; Sigma, St. Louis, MO) and fixed in a Kopf small stereotaxic device with hollow ear bars.

In experiment I, blood samples were drawn at the conclusion of each sound stimulation and recording session. In experiment II, blood samplings were performed concurrently with sound stimulation and recording. Birds were tracheotomized before being placed in a Kopf device with the ventral surface of the brain exposed, allowing both recordings and blood samplings from the ventral surface. 


\begin{tabular}{|c|c|c|c|c|}
\hline Stimulus & Excitatory & Inhibitory & No response & Total units \\
\hline \multicolumn{5}{|c|}{ Preoptic area (POA), 334 units recorded from 14 birds } \\
\hline Female nest coo & $32(19.8 \%)$ & $17(10.7 \%)$ & $114(71.7 \%)$ & 163 \\
\hline Male nest coo & $9(8.8 \%)$ & $11(10.8 \%)$ & $82(80.4 \%)$ & 102 \\
\hline Reversed-FNC ${ }^{a}$ & $1(2.8 \%)$ & $1(2.8 \%)$ & $34(94.4 \%)$ & 36 \\
\hline Reversed-MNC ${ }^{b}$ & $0(0.0 \%)$ & $1(5.9 \%)$ & $16(94.1 \%)$ & 17 \\
\hline White noise & $0(0.0 \%)$ & $0(0.0 \%)$ & $16(100.0 \%)$ & 16 \\
\hline \multicolumn{5}{|c|}{ Anterior medial hypothalamus (AMH), 346 units recorded from 12 birds } \\
\hline Female nest coo & $41(23.5 \%)$ & $22(12.6 \%)$ & $116(66.7 \%)$ & 179 \\
\hline Male nest coo & $16(14.2 \%)$ & $21(18.5 \%)$ & $76(67.3 \%)$ & 113 \\
\hline Reversed-FNC & $0(0.0 \%)$ & $1(4.2 \%)$ & $23(95.8 \%)$ & 24 \\
\hline Reversed-MNC & $1(5.3 \%)$ & $1(5.3 \%)$ & $17(89.4 \%)$ & 19 \\
\hline White noise & $0(0.0 \%)$ & $0(0.0 \%)$ & $11(100.0 \%)$ & 11 \\
\hline \multicolumn{5}{|c|}{ Ventromedial nucleus (VMN), 255 units recorded from 10 birds } \\
\hline Female nest coo & $6(6.8 \%)$ & $8(9.1 \%)$ & $74(84.1 \%)$ & 88 \\
\hline Male nest coo & $4(4.7 \%)$ & $12(14.0 \%)$ & $70(81.3 \%)$ & 86 \\
\hline Reversed-FNC & $2(5.6 \%)$ & $4(11.1 \%)$ & $30(83.3 \%)$ & 36 \\
\hline Reversed-MNC & $1(2.9 \%)$ & $3(8.8 \%)$ & $30(88.3 \%)$ & 34 \\
\hline White noise & $0(0.0 \%)$ & $1(9.1 \%)$ & $10(90.9 \%)$ & 11 \\
\hline
\end{tabular}

${ }^{a}$ Reversed female nest coo.

${ }^{b}$ Reversed male nest coo.

Acoustic stimuli. Acoustic stimuli used in this study were as follows: (1) female nest coo, (2) male nest coo, (3) reversed female nest coo, (4) reversed male nest coo, and (5) male bow coo (an agnostic coo emitted only by males, used in a few trials). To obtain the stimuli, multiple coo vocalizations were recorded from 16 different individuals, using a Marantz PMD 430 stereo cassette tape recorder. Recordings were made with a Sennheiser microphone placed 4 inches above the nest bowl and connected to the tape recorder outside the acoustic isolation chamber. The behavioral context of each coo recorded was observed and noted. Manipulation and analysis of vocalizations using a computerized analysis and resynthesis system have been described (Margoliash, 1983; $\mathrm{Yu}$ and Margoliash, 1996). Analog recordings of the nest coos were digitized at a sampling rate of $22.6 \mathrm{kHz}$ at 16 bit resolution on a Gateway (North Sioux City, SD) 2000 486/33 computer equipped with a DT 2821 analog-to-digital board (Data Translation, Marlboro, MA) and displayed spectrographically on the computer screen using SIGNAL sound analysis software, version 2.23 (Engineering Design, Belmont, MA). Reversed coos were created using the "reverse time buffer" command. Computergenerated $500 \mathrm{msec}$ white noise bursts [two/sec at $75 \mathrm{~dB}$ sound pressure level (SPL)] with a bandwidth of $0-11 \mathrm{kHz}$ were used. All vocalizations were standardized to an intercall interval of $2 \mathrm{sec}$ and to a maximum amplitude of $75 \pm 2 \mathrm{~dB}$ SPL. The tape of a female's nest coos was edited to contain a high number of coos without noise or other artifacts. Each session lasted $280 \mathrm{sec}$, with a between-sessions pause of $60 \mathrm{sec}$. Tapes of a male nest coo, a reversed female nest coo, a reversed male nest coo, and white noise $(100 \mathrm{sec})$ were similarly edited. In addition, a composite tape was created consisting of three parts in order: white noise (100 sec), female nest coo $(280 \mathrm{sec})$, and reversed female coo $(100 \mathrm{sec})$, with a 60 sec interval between parts. When recording from the ventromedial nucleus (VMN), bow coo stimulation was added. The edited but undigitized tape of coos was used when tracking responsive units. Once a responsive unit was located, we switched to the computer resynthesized recordings for further study of the response properties of the unit. Each coo was presented 10-12 times, with 4-6 sec between trials. When the response properties of the same unit to different coos were studied, different sounds were presented in random sequences and repeated 10-20 times. For the 22 birds from which we also collected blood samples, a single stimulus was presented.

Sounds were presented via either a Yamaha NS 10M loudspeaker placed in front of the birds $(n=6)$ at a distance of $\sim 80 \mathrm{~cm}$ or a Sony earphone (diameter, $3.5 \mathrm{~cm}$ ) within a cone-shaped box connected to the hollow ear bars. The loudspeaker was calibrated in decibels SPL using a calibrated condenser microphone (Bruel-Kjaer 4135) placed to duplicate the position of the bird's head in the stereotaxic instrument. The output of the earphones was calibrated with a $1 / 2$ inch condenser microphone
(Bruel-Kjaer 4135) attached to a funneled probe that was inserted into the opening of each hollow ear bar through a small canal at its center (Biederman-Thorson, 1970a,b). The peak amplitude of the sound stimuli was set to between 70 and $75 \mathrm{~dB}$ sound pressure level with a manual attenuator. The outputs of different acoustic stimuli were monitored by the storage oscilloscope.

Recordings. Extracellular recordings were made from single cells located within the preoptic area (POA), the anterior medial hypothalamus $(\mathrm{AMH})$, or the VMN. Single cells were recorded with glass micropipettes (tip, 1-3 $\mu \mathrm{m} ; 6-15 \mathrm{M} \Omega$ impedance by BL-1000B microelectrode tester) filled with $2 \%$ potamine sky blue (PSB; ICN Biomedicals, Cleveland, $\mathrm{OH}$ ) in $3 \mathrm{M} \mathrm{NaCl}$. In experiment I, recording microelectrodes were inserted into the POA, $\mathrm{AMH}$, or $\mathrm{VMN}$ from the dorsal plane with a Burleigh Piezoelectric Microstepper. Coordinates for placement of recording electrodes were as follows: POA, anterior, $4.8-5.2 \mathrm{~mm}$; lateral, 0.6-0.8 $\mathrm{mm}$; and ventral, 7.5-8.0 mm; $\mathrm{AMH}$, anterior, $3.5-4.0 \mathrm{~mm}$; lateral, $0.5-0.8 \mathrm{~mm}$; and ventral, $7.9-8.3 \mathrm{~mm}$; and $\mathrm{VMN}$, anterior, $2.6-3.0 \mathrm{~mm}$; lateral, $0.4-0.7 \mathrm{~mm}$; and ventral, $8.0-8.4 \mathrm{~mm}$. In experiment II, the recording microelectrodes were lowered at a $5-8^{\circ}$ angle from the vertical line at an entry point $1-1.5 \mathrm{~mm}$ lateral to the midline of the optic chiasm to POA or AMH under the visual guidance of a Zeiss operation microscope. Micropipettes were connected to a preamplifier by a 0.12 $\mathrm{mm}$ wire of platinum lead. Single-unit signals were amplified by a Neurodata IR-283 preamplifier, monitored with a Marantz 420 audio monitor, and displayed together with acoustic stimuli on a Tektronix 5110 storage oscilloscope. A Nicolet 4094C digital oscilloscope was linked to a Macintosh IIx computer via a National Instrument NB-GPIB board for collection on-line in the form of continuous rate meter and perievent histogram records.

Records were stored on magnetic tape for off-line analysis by the software program SCOPE (courtesy of J. Tepper, Rutgers University), using a Macintosh IIx computer and a National Instrument NB-M I0 16L multifunction board. The neurons responding to acoustic stimulus were studied and classified by the poststimulus and peristimulus time histogram. The number of spikes evoked by each stimulus was recorded for the duration of the stimulus, and the series was repeated at $75 \pm 2 \mathrm{~dB}$ SPL for each unit. The spikes were counted digitally. A response was considered excitatory when the firing rate during an acoustic stimulus was increased at least $15 \%$ above the control levels of background firing; a response was considered inhibitory when spontaneous discharge was depressed at least $15 \%$ by an acoustic stimulus. The spontaneous spike rate was measured 5-10 sec before the onset of an acoustic stimulus. The stimulus spike rate was defined as the number of spikes produced during the stimulus divided by the duration of the stimulus.

Blood sampling procedure. Birds were anesthetized with urethane 


\section{Preoptic Area}

\section{A Female Nest Coo}

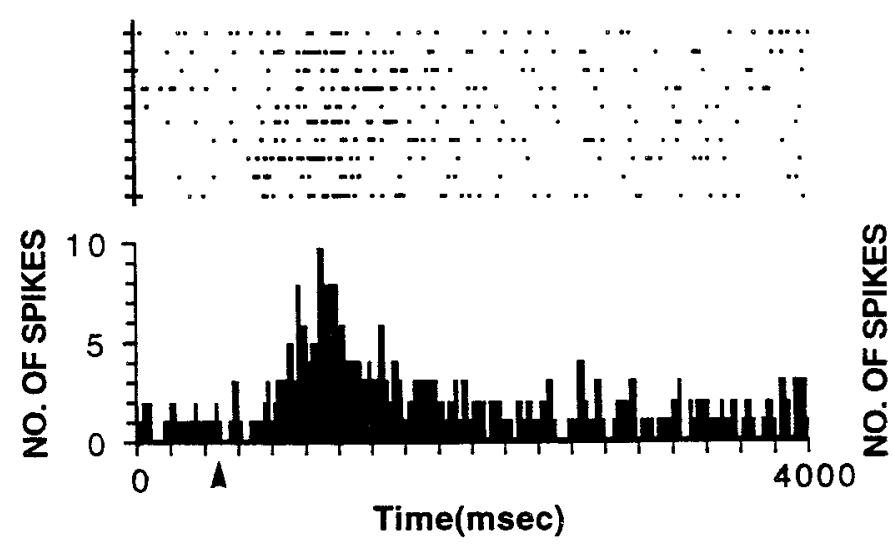

B Reversed Female Nest Coo
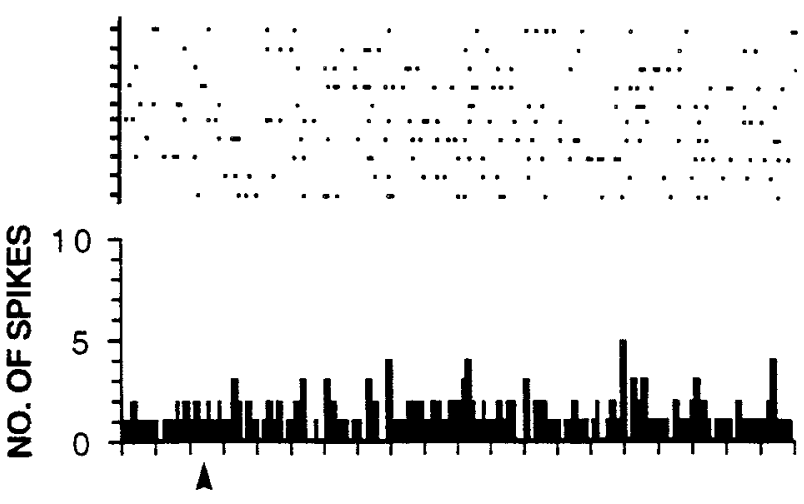
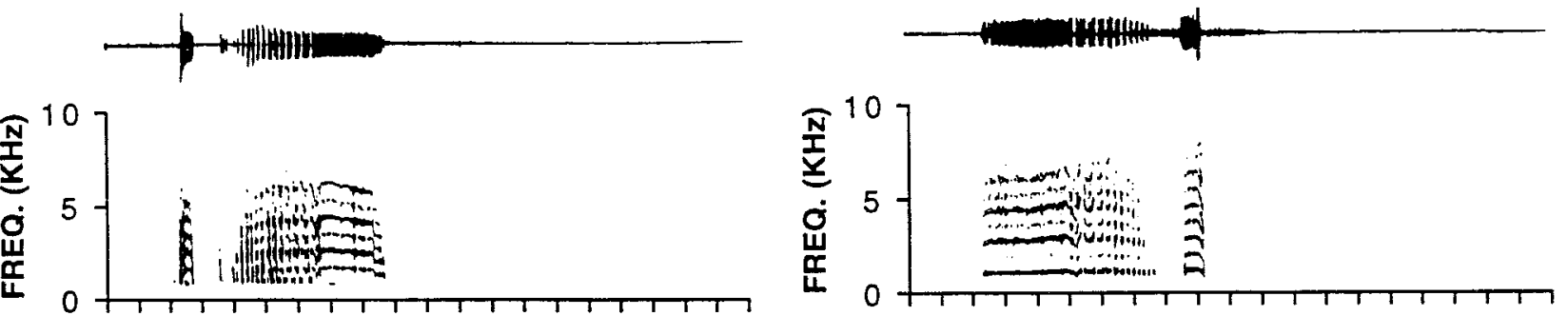

C White noise
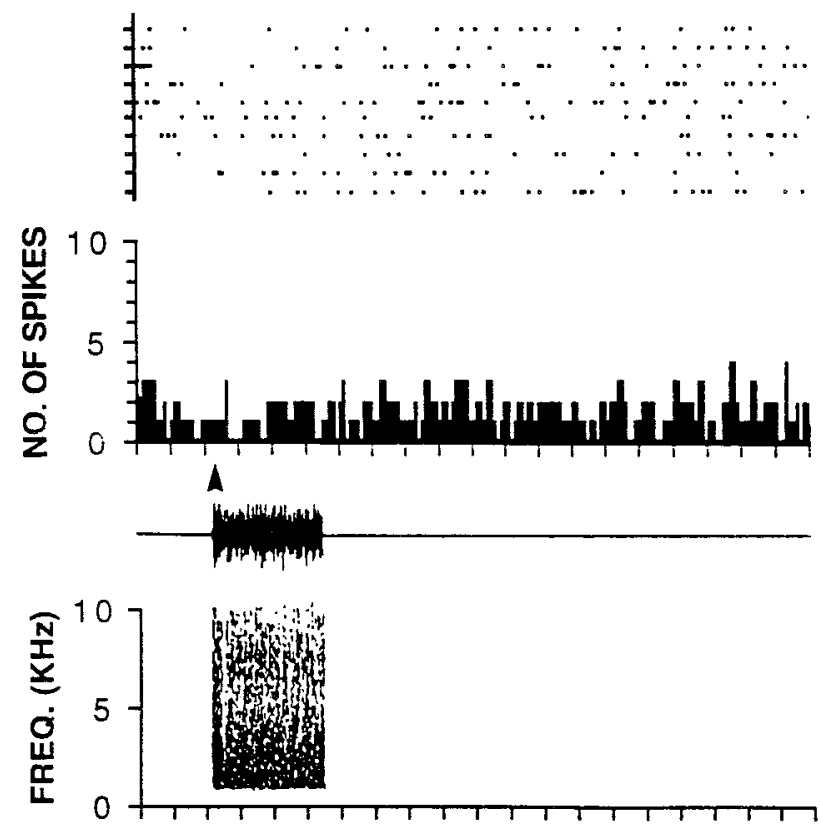

D Male Nest Coo
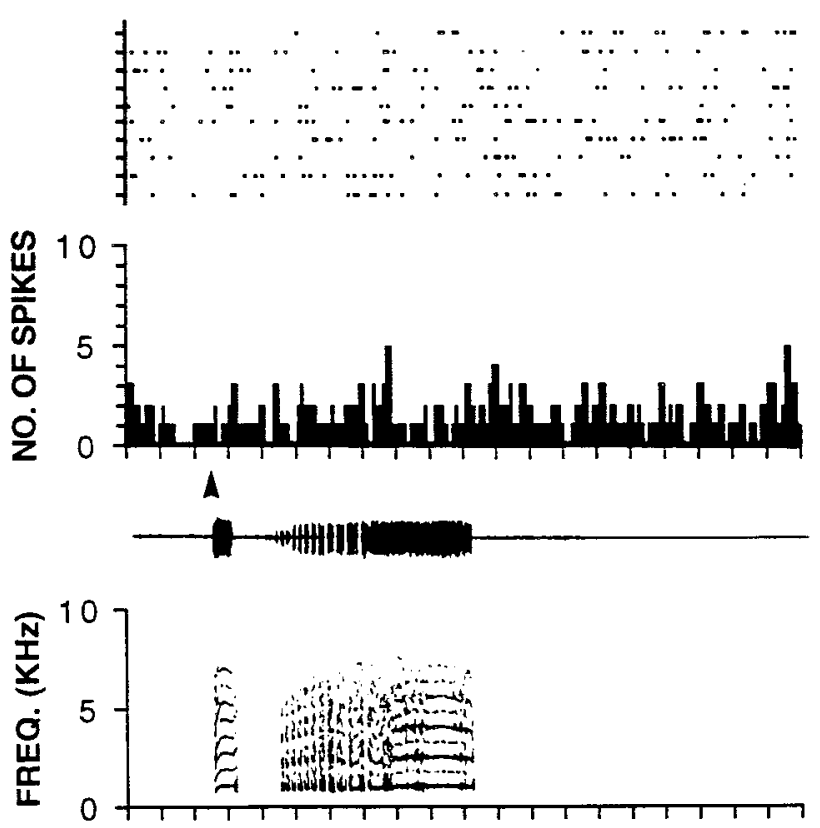

Figure 2. Response of unit 960108 in the preoptic area of a female ring dove to different acoustic stimuli. $A$, The female nest coo as the stimulus; the activity of the unit increases significantly. Top, Dot raster plot showing 10 sweeps of the unit's response. Each dot represents one spike. Bin size is 20 msec. Middle, Histogram of the unit's response. Bottom, Computer amplitude display and spectrogram of the female nest coo: amplitude over time. $B$, Reversed female nest coo as the stimulus; no change in neuronal activity can be detected in the number of spikes over time or in the histogram representation. $C$, White noise as the stimulus: no change in neuronal activity. $D$, Male nest coo as the stimulus: no change in neuronal activity. All histograms in this paper represent the sum of 10 repetitions of the stimulus. All units are well isolated. In all figures, a closed triangle denotes the onset of the stimulus. 


\section{Ventromedial Nucleus}

\section{A Female Nest Coo}
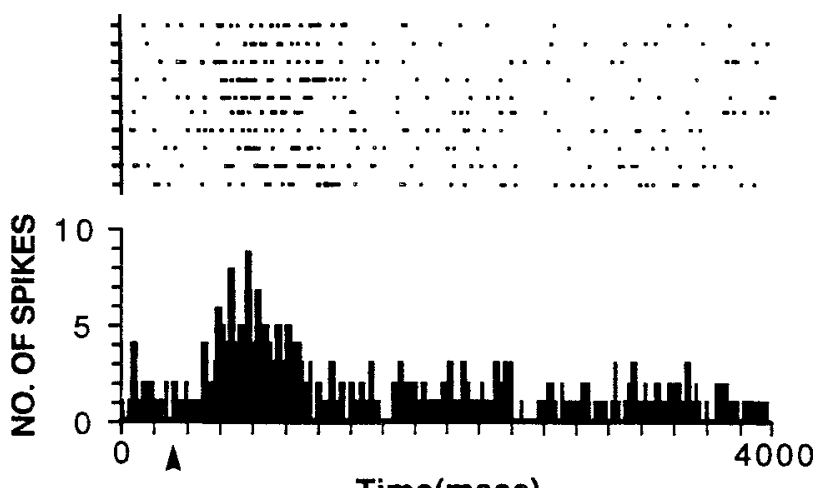

Time(msec)

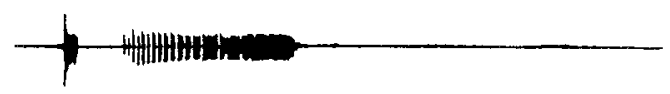

B Male Bow Coo
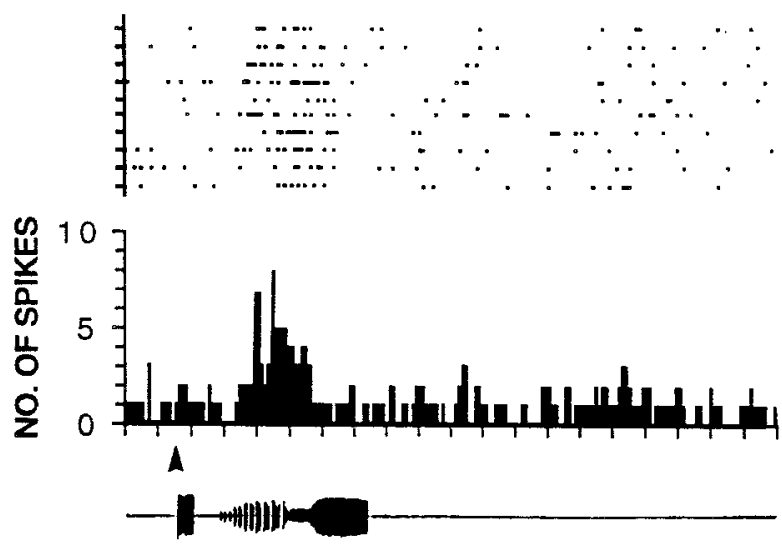

\section{Male Nest Coo}
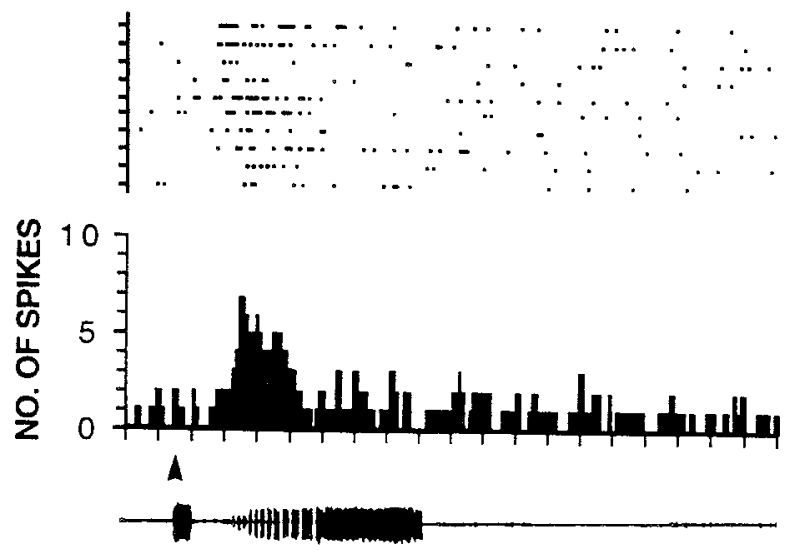

Figure 3. Response of excitatory unit 971216 to different coo stimuli in the ventromedial nucleus. $A$, The female nest coo. Top, dot raster plot of the unit's response; middle, histogram of the unit's response; bottom, computer amplitude display of the female nest coo. $B$, Male bow coo. $C$, Male nest coo; note strong firing response to the female nest coo, relatively weaker response to the male nest coo, and intermediate response to the male bow coo.

\section{Preoptic Area}

\section{FNC-Specific Unit}
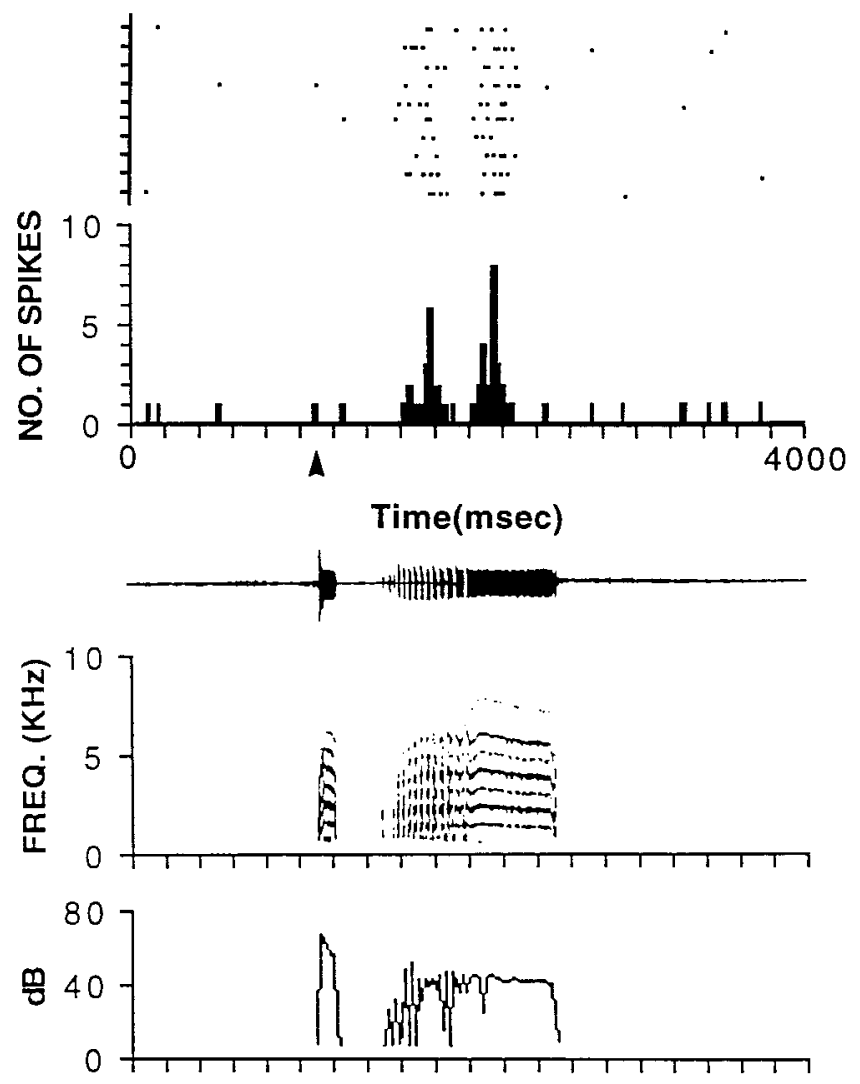

Figure 4. Response of female-nest-coo-specific unit 960107 in the preoptic area of a female ring dove. Top, Dot raster plot and histogram showing response of the unit to the female nest coo presented at $70 \pm 5$ dB SPL. Bottom, Computer amplitude display and spectrogram of the female nest coo.

$(0.4 \mathrm{ml} / \mathrm{kg}$, i.m. $)$. The procedure used in withdrawing blood from the pituitary veins has been described (Huang et al., 1995; Peng et al., 1996). In brief, the pituitary gland and the ventral diencephalon were exposed parapharyngeally under the visual guidance of a Zeiss dissecting microscope. Using a glass cannula with a polyethylene tube, blood was drained from the veinal region of the pituitary gland and collected into a vessel at 10-20 min intervals for a duration of $120 \mathrm{~min}$. Blood samples were immediately centrifuged at $10,000 \times g$ for $1 \mathrm{~min}$. Serum samples were stored at $-20^{\circ} \mathrm{C}$ until radioimmunoassay (RIA) for $\mathrm{LH}$. In experiment I, blood samplings at 10-20 min intervals for 120 min commenced after the sound stimulation and recording session, which lasted 50-70 min. The pituitary gland was surgically exposed immediately after freeing the bird from the recording position. This procedure usually took $15-25 \mathrm{~min}$. In experiment II, blood samples were collected at 10-20 min intervals for $120 \mathrm{~min}$ while the recording and sound stimulation were in progress; recording and sound stimulation were allowed to run for $40-50 \mathrm{~min}$ before the first sampling was performed.

Localization of recording electrodes. The position of each recording electrode was marked by iontophoresis of PSB by passing 5-10 $\mu \mathrm{m}$ negative current for $10-20 \mathrm{~min}$ through the recording electrode or by application of PSB dyes using a pneumatic pressure ejection system (Cheng and Peng, 1997). The anesthetized birds were intracardially perfused with $0.9 \% \mathrm{NaCl}$ solution followed by $10 \%$ formalin. All recording sites were localized by examination of $40-60 \mu \mathrm{m}$ frozen sections (Fig. 1). After perfusion, the birds were laparotomized to determine the size of the largest follicles. Only birds with follicles of F5 and F4 were used for the study. F4 and F5 represent the lower end of preovulatory follicles 
Table 2. Breakdown of excitatory responses to female nest coos in three regions of the hypothalamus

\begin{tabular}{lllr} 
& \multicolumn{2}{l}{$\begin{array}{l}\text { No. }(\%) \text { of units exhibiting } \\
\text { excitatory responses }\end{array}$} \\
\cline { 2 - 4 } & FNC-specific & Other & Total \\
\hline Preoptic area (POA) & $4(12.5 \%)$ & $28(87.5 \%)$ & 32 \\
Anterior medial hypothalamus & & & \\
$\quad(\mathrm{AMH})$ & $5(12.2 \%)$ & $36(87.8 \%)$ & 41 \\
Ventromedial nucleus (VMN) & $0(0.0 \%)$ & $6(100.0 \%)$ & 6
\end{tabular}

$\overline{{ }^{a} \text { Female-nest-coo-specific units are silent or near-silent units that respond selectively }}$ to playbacks of the female nest coo and exhibit a characteristic pattern of two bursts separated by a period of slow spiking or complete silence. One such unit in the AMH exhibited only a single burst.

within the hierarchy, with F1 nearest to the time of LH surge (Bahr et al., 1983). By selecting birds with small follicles and therefore low LH output, we allowed a greater latitude for the system to exhibit any incremental changes of $\mathrm{LH}$ output.

LH radioimmunoassay. Plasma $\mathrm{LH}$ was assayed by means of an RIA specific for chicken LH (PRC-AE1-1; Scanes and Follett, 1972) obtained from Dr. Peter Sharp (Agriculture and Food Research Council Poultry Research Center, Roslin, Midlothian, UK). The assay was performed in the laboratory of P. Johnson (Cornell University). The antiserum, also obtained from Dr. Sharp, was designated anti-chicken LH 3/3 (Sharp et al., 1987). Before assay of the experimental samples, ring dove plasma was tested at various dilutions to verify parallelism in the assay. The mean interassay CVs for high and low pools included in our assays were 10.3 and $12.9 \%$, respectively.

\section{RESULTS}

Of the 24 birds in experiment I, 14 females were used exclusively for recording and sound stimulation. Blood samplings, 10 for each bird, were attempted for the remaining 10 birds. Samplings for eight birds were successful; the other two were aborted. Blood samplings were completed for all 12 birds in experiment II. In total, RIA was performed on the 22 birds ( 20 females and 2 males) for which 10 complete samplings were taken. One bird with four samplings was not included in the statistical treatment.

Table 1 provides a summary of the neuronal responses recorded from units in the POA, AMH, and VMN (also known as the posterior medial hypothalamus) in response to different acoustic stimuli, including white noise. Extracellular recordings

\section{Ant. Med. Hypoth.}

\section{FNC-Specific Unit}

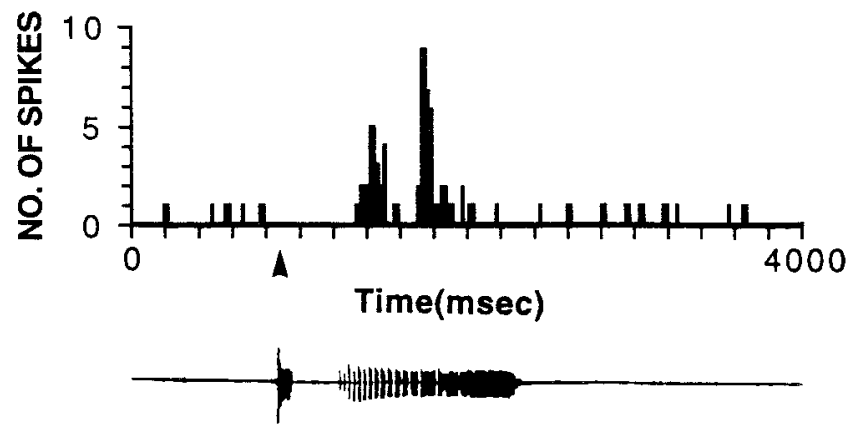

Figure 5. Response of female-nest-coo-specific unit 960127 in the anterior medial hypothalamus (Ant. Med. Hypoth.). Histogram shows the unit's response to the female nest coo stimulus presented at $70 \pm 5 \mathrm{~dB}$ SPL.

\section{Ant. Med. Hypoth.}

\section{FNC-Specific Unit}
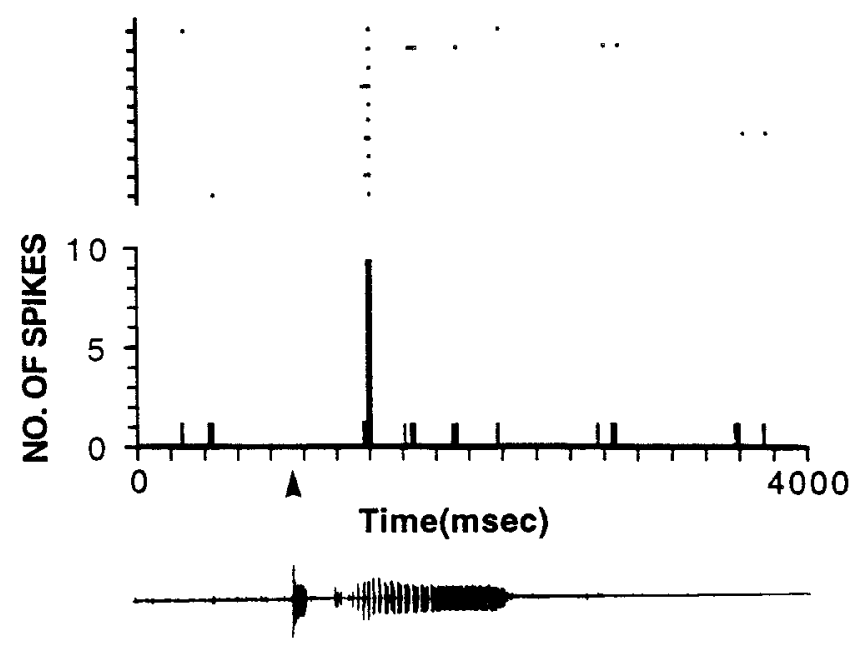

Figure 6. Unique response of anterior medial hypothalamus (Ant. Med. Hypoth.) unit 960124 to the female nest coo. Top, Dot raster plot showing the unit's single spike response. Middle, Histogram of the unit's response. Bottom, Computer amplitude display of the female nest coo.

were made from 334 spontaneously firing neurons in POA, 346 in $\mathrm{AMH}$, and 225 in VMN. Figure 1 shows the distribution of recording sites in these nuclei. In POA, as well as in $\mathrm{AMH}$ and VMN, some neurons exhibited a fast, regular pattern of discharge averaging $6.2 \pm 1.2 \mathrm{impulses} / \mathrm{sec}$; others showed a slow and irregular pattern of discharge averaging $3.7 \pm 0.6 \mathrm{impulse} / \mathrm{sec}$; and still others showed a phasic discharge pattern.

\section{Effects of female nest coos on neuronal activity}

The following results were based on analyses of 199 units in the POA, 203 in the AMH, and 124 in the VMN.

\section{Excitatory responses}

Within the POA, $20 \%(n=32)$ of the units recorded exhibited excitatory responses to the female nest coo. The average firing rate increased significantly from the baseline rate of $5.6 \pm 0.8$ to $12.4 \pm 1.1$ (mean $\pm \mathrm{SD})$ spikes/sec $(p<0.01)$. The excitatory response exhibits one interesting feature unique to units of this group: the increased firing rate was not a continuous train of discharge; it was interrupted by a period ( $300 \mathrm{msec})$ of a nearbaseline firing rate during a long response bout lasting 1200-1600 msec. This bursting pattern is most striking in a few units we designated as female-nest-coo-specific units (see below). Of 32 excitatory-responsive neurons, 29 neurons selectively responded to the female nest coo. Figure 2 illustrates one such neuron. Figure $2 A$ shows a significant increase in discharge in response to the female nest coo $(p<0.01)$; note the baseline firing in the middle of response. Figure $2 B$ shows no response to the reversed female nest coo. Figure $2 C$ shows insignificant changes in response to white noise, and Figure $2 D$ shows insignificant changes in response to the male nest coo. The other three neurons showed weak excitatory discharge in response to each of the four stimuli (not significantly different from baseline rate; $p>0.05$ ).

Within the AMH, 24\% $(n=41)$ of the recorded units exhibited 


\section{Preoptic Area}

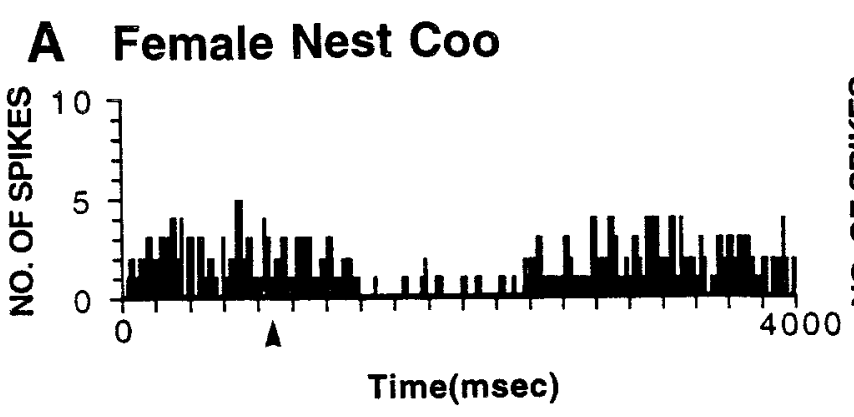

B Reversed Female Nest Coo

\section{White noise}

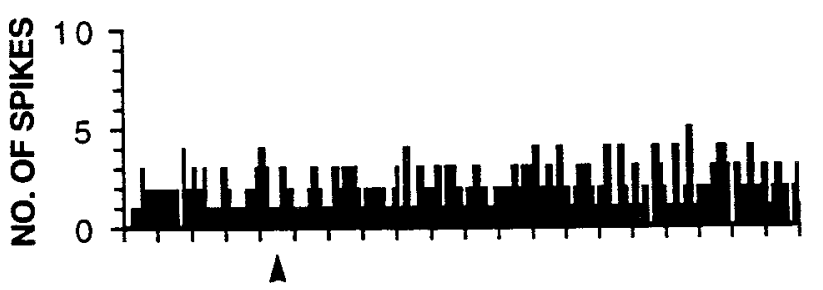

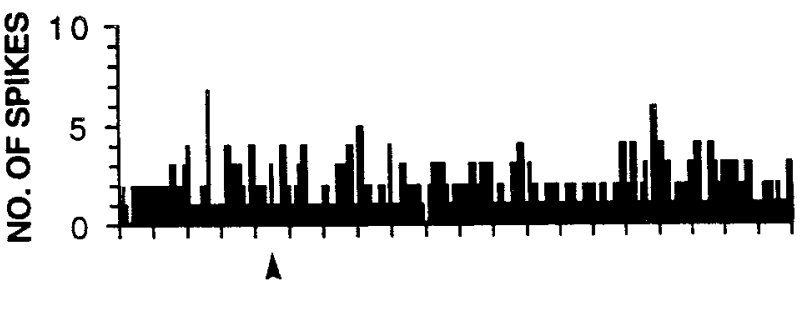

D Male Nest Coo

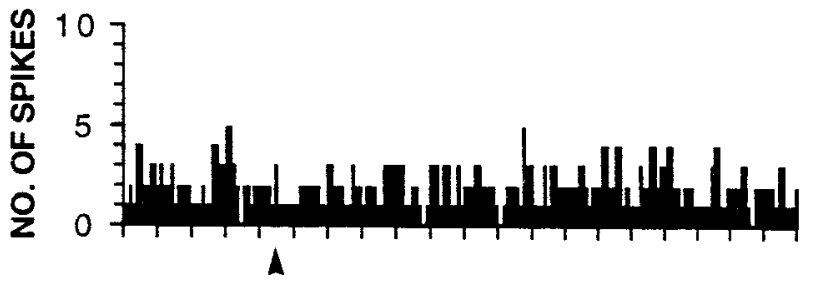

Figure 7. Response of inhibitory unit 960126 in the preoptic area to different acoustic stimuli in a female ring dove. $A$, Histogram shows the unit's response. With the female nest coo as stimulus, the unit's response declines significantly. $B-D$, Unit's response, respectively, to reversed female nest coo, white noise, and the male nest coo. None of these stimuli evoked changes in neuronal activity.

excitatory responses to the female nest coo. The average firing rate increased significantly from the baseline rate of $4.4 \pm 0.7$ to $9.5 \pm 1.2$ spikes/sec $(p<0.01)$. With the exception of five neurons that also exhibited a significant increase of discharge in response to male nest coos, these excitatory neurons $(n=36)$ showed a selective response to female nest coos. In comparison with female-nest-coo-sensitive excitatory neurons in the POA, the responses of the AMH neurons were 200-250 msec longer in duration. Most AMH neurons displayed a continuous discharge without an episode of the silent or near-baseline discharge characteristic of POA neurons; eight AMH neurons exhibited the POA type of discharge.

As in the POA and $\mathrm{AMH}$, there were units in the VMN in which female nest coo stimulation evoked an excitatory response, but they were fewer in number (six units, or $6.8 \%$ of 88 neurons). The average firing rate of such units increased significantly from the baseline rate of $5.6 \pm 0.6$ to $12.4 \pm 1.2 \mathrm{spikes} / \mathrm{sec}(p<0.01)$ with a duration comparable to that of a female nest coo bout. Four of these six units also exhibited significant excitatory responses to male nest coo and male bow coo stimulation $(p<$ 0.05 ). Figure 3 shows discharge patterns of unit 971216 to three different coos. The discharge evoked by female nest coo stimulation was the strongest (Fig. $3 A$ ), followed by that evoked by the male bow coo (Fig. $3 B$ ) and then the male nest coo (Fig. 3C), all of which showed a significant change from the baseline rate.

\section{Female-nest-coo-specific units}

Four excitatory units in the POA and five in the AMH exhibited a discharge pattern distinct from the female-nest-coo-sensitive excitatory units described in the preceding sections. These units typically were silent $(n=6)$ or displayed slow and irregular discharges $(n=3)$, with a discharge train of 7-15 spikes and a response latency of $400-550 \mathrm{msec}$ to female nest coo stimulation.
Figure 4 shows the evoked response of one such neuron (960107) in the POA to female nest coo stimulation.

The spike train of neurons that responded only to the female nest coo consisted of two bursts, of four to seven spikes each, separated by a silent period of 200-600 msec. The pattern approximately corresponds temporally to the two-note coo. This discharge pattern was evoked exclusively by the female nest coo; neither the male nest coo nor the reversed female nest coo induced a two-part spike train. In the present experiments, these neurons were normally silent or near silent when not responding to the female nest coo. Because of these unique features, we designated them female-nest-coo-specific neurons. Table 2 shows the number of female-nest-coo-specific units recorded in the three regions of the hypothalamus. It should be noted that such neurons recorded from AMH did not always exhibit the distinct two-burst pattern; the response latency $(350-550 \mathrm{msec})$ was comparable to that of POA units (Fig. 5). One unique silent neuron in the AMH exhibited a single spike response to female nest coo stimulation (Fig. 6). We have not found any female-nest-coo-specific units in the posterior medial hypothalamus.

\section{Inhibitory response}

Some of the female-nest-coo-sensitive neurons exhibited an inhibitory response. A total of 17 units in the POA showed a decline of firing rate on average from $6.7 \pm 0.9$ to $3.1 \pm 0.6$ spikes/sec $(p<0.01)$. Of these, eight units showed a high spontaneous firing rate $(11.2 \pm 1.11$ spikes/sec), seven units showed a phasic discharge, and two units showed a slow and irregular discharge. Fourteen inhibitory units were selectively responsive to the female nest coo. Figure 7 shows one such neuron: an inhibitory response to the female nest coo (Fig. $7 A$ ) but virtually no change in firing rate in response to the reversed female nest coo or white noise or male nest coo (Fig. $7 B-D$ ). Three neurons showed an 


\section{Preoptic Area}

\section{A Male Nest Coo}
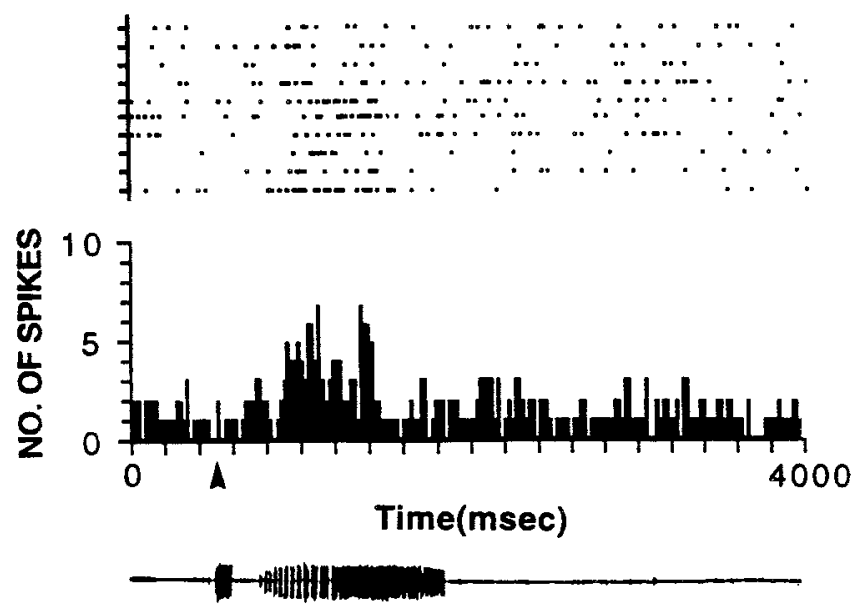

B Male Nest Coo
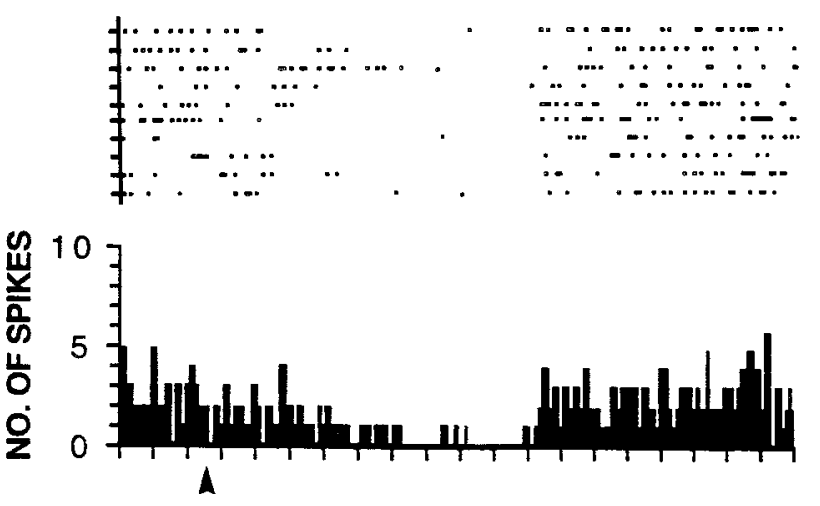

Figure 8. Response of units in the preoptic area to the male nest coo. $A$, Excitatory response. Top, Dot raster plot of the unit's response; middle, histogram of the response; bottom, computer amplitude display of the male nest coo. $B$, Inhibitory response. Top, Dot raster plot of the unit's response; middle, histogram of the response; bottom, computer amplitude display of the male nest coo.

inhibitory response to the female nest coo and also showed a weak (statistically insignificant, $p>0.05$ ) inhibitory response to male nest coos and reversed female nest coos.

In the AMH, 22 units exhibited inhibitory responses to female nest coo stimulation. On average, the firing rate declined significantly, from $5.3 \pm 0.8$ to $2.4 \pm 0.5$ spikes/sec. Five of these units also showed inhibitory responses $(p<0.02)$ to the male nest coo and reversed female nest coo. In the VMN, eight units showed inhibitory responses to female nest coo stimulation. On average, the firing rate declined from $5.8 \pm 0.8$ to $3.1 \pm 0.6$. Of these units, three showed phasic discharge, one showed a fast discharge, and one showed a slow and irregular discharge. Only three units showed selective inhibitory responses to female nest coo stimulation.

Most recorded units showed no response to female nest coo stimulation (Table 1). In total, 114 neurons in the POA, 116 in the $\mathrm{AMH}$, and 74 in the $\mathrm{VMN}$ did not respond to female nest coo stimulation.

\section{Ant. Med. Hypoth.}

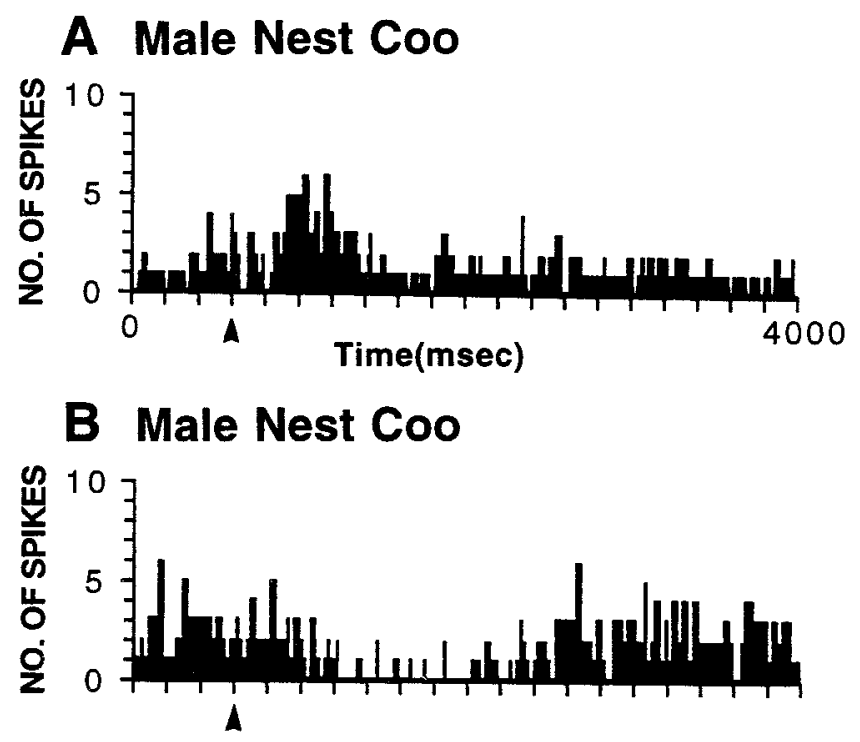

Figure 9. Response of units in the anterior medial hypothalamus (Ant. Med. Hypoth.) to the male nest coo in a female ring dove. A, Histogram showing response of excitatory unit 960107. B, Histogram showing response of inhibitory unit 960915 .

\section{Effects of reversed female nest coos on neuronal activity}

The response to reversed female nest coos contrasts starkly with the response to normal female nest coos. We recorded only one unit in the POA-AMH region that exhibited a weak excitatory response to reversed female nest coos, with the firing rate increasing from $4.2 \pm 0.3$ to $5.5 \pm 0.5$ spikes/sec $(p>0.05)$. However, six units in the VMN showed significant responses to the reversed female nest coo; two were excitatory and four were inhibitory.

\section{Effects of male nest coo on neuronal activity}

Responses to male nest coo stimulation were recorded from a total of 102 units in the POA, 113 in the $\mathrm{AMH}$, and 86 in the VMN. Nine units in the POA showed excitatory responses to the male nest coo with an average firing rate increase of $4.9 \pm 0.5$ to $8.7 \pm 0.8$ spikes/sec (Fig. $8 A$ ). Seven of these units did not respond when the male nest coo was reversed, and two showed a response to the female nest coo as well $(5.4 \pm 0.3$ to $6.5 \pm 0.7$ spikes/sec). Responses of 11 units were inhibitory, with the average firing rate declining from $5.8 \pm 0.6$ to $3.2 \pm 0.4$ spikes $/ \mathrm{sec}$ (Fig. $8 B$ ). Eight of these units showed no change in discharge when reverse male nest coos were presented, and the remaining three units showed a decline in discharge $(p<0.05)$.

Sixteen units in the AMH showed excitatory responses, with an average increased firing of $5.1 \pm 0.8$ to $11.4 \pm 1.2$ spikes/sec (Fig. $9 A)$. Five of these also showed excitatory responses to other acoustic stimuli $(p<0.05)$. Responses of 21 units were inhibitory, with an average decline from $6.5 \pm 0.7$ to $2.7 \pm 0.6$ spikes $/ \mathrm{sec}$ (Fig. $9 B$ ). Seven of these units also showed various degrees of inhibitory responses to female nest coos, reversed male nest coos, and reversed female nest coos.

In the VMN, 4 units showed excitatory responses and 12 units showed inhibitory responses. Most of these units were not selectively responsive to the male nest coo. We did not find units 
A
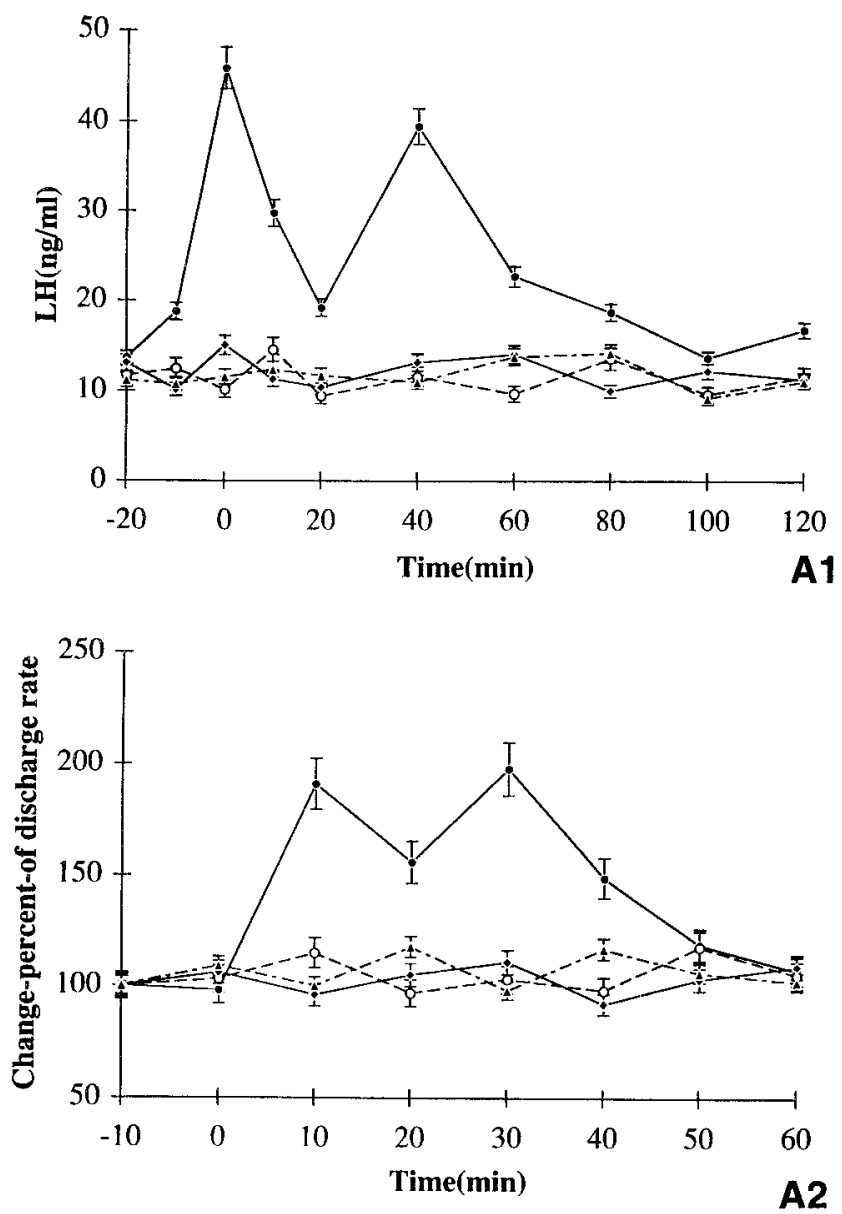

B

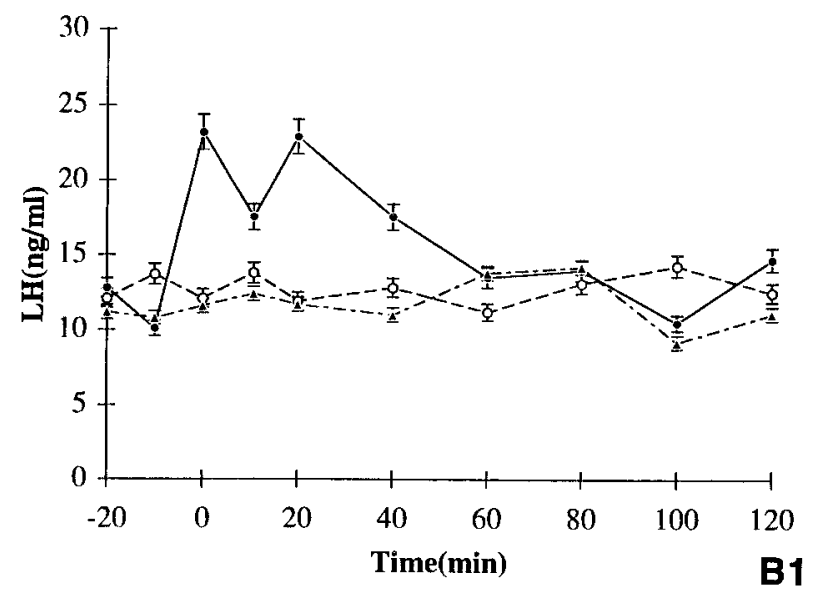

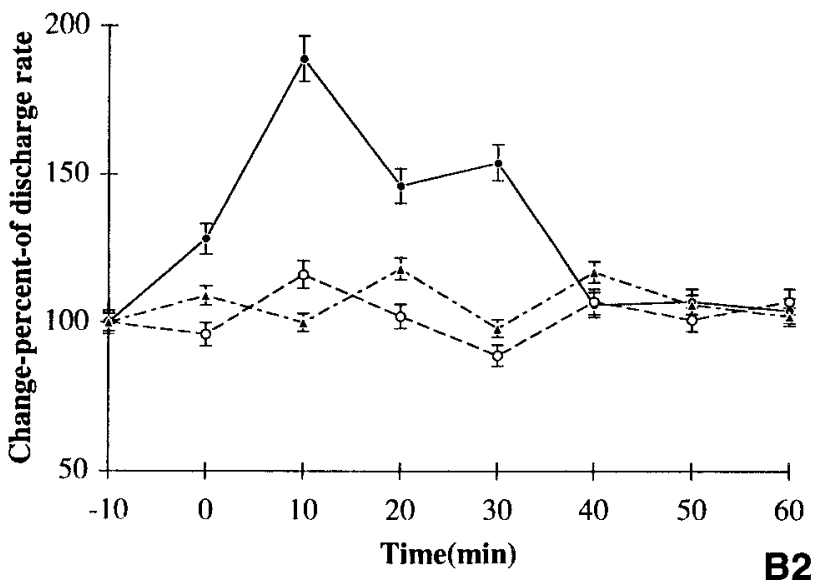

Figure 10. Effects of nest coo stimulation on neuronal response of preoptic area units and pituitary LH levels in female ring doves. There is a significant effect of different acoustic stimuli on LH levels $\left(F_{(5,54)}=9.86 ; p<0.01\right)$ and on percentage discharge rate $\left(F_{(5,42)}=4.57 ; p<0.01\right) . A$, Effects of the female nest coo. A1, LH levels were significantly higher for the female birds $(n=3)$ hearing female nest coo playbacks (closed circles) than for those $(n=2)$ receiving no playback (closed triangles) at time points 0,10 , and $20 \mathrm{~min}$ in which $0=40 \mathrm{~min}$ after the onset of female nest coo stimulation $(t=3.68 ; p<0.01)$. Hearing the reversed female nest coo (open circles; $n=2)$, on the other hand, produced LH levels indistinguishable from those of the control $(t=0.44 ; p>0.05)$. A2, Percentage changes of firing rate of units recorded during each sound stimulation. Closed circles. Responses of six units responsive to the female nest coo; closed triangles, responses of four units $(n=2)$ when there was no playback; open circles, responses of four units to reversed female nest coo stimulation; closed rhombuses, responses of three units to white noise stimulation. $B$, Effects of the male nest coo. B1, LH levels after hearing the male nest coo (closed circles) were greater than in the control (closed triangles) $(t=2.31 ; p<0.05)$. Reversing the male nest coo (open circles) produced no changes in LH response $(t=1.53 ; p>0.05)$. A comparison of LH levels by female nest coo and male nest coo stimulation showed a significant difference $(t=2.31 ; p<0.05)$. B2, Percentage changes of firing rate of units recorded during each sound stimulation. Closed circle, male nest coo responses of four units; open circles, reversed male nest coo responses of three units. Note that the percentage changes of neuronal response to the female nest coo (Fig. 10A2) and male nest coo (Fig. 10B2) are similar $(t=0.65 ; p>0.05)$.

whose response features met the criteria to be designated as female-nest-coo-specific neurons.

\section{Effects of nest coo stimulation on pituitary LH level}

LH levels in 220 blood samples from 19 experimental birds and 3 control birds provided the basis for analysis. Ten samples were taken from each bird, the first five at $10 \mathrm{~min}$ intervals and the second five at $20 \mathrm{~min}$ intervals. The control birds underwent identical surgical and recording procedures but without sound stimulation.

Five different coos (female nest coo, reversed female nest coo, male nest coo, reversed male nest coo, and male bow coo) and white noise were used as acoustic stimuli. The average LH concentration in the pituitary was significantly higher in response to the female nest coo $(24.62 \pm 3.12 \mathrm{ng} / \mathrm{ml})$ than to any other acoustic stimulus (male nest coo, $15.25 \pm 2.31 \mathrm{ng} / \mathrm{ml}$; white noise, $13.65 \pm 2.41 \mathrm{ng} / \mathrm{ml}$; control, $11.49 \pm 2.57 \mathrm{ng} / \mathrm{ml} ; p<0.01)$. All three nuclei received the same set of stimuli, but a high LH level was observed only when POA or AMH units were activated in response to female nest coo stimulation. Figure 10 illustrates the neuronal activity of one POA neuron in response to the female nest coo, reversed female nest coo, male nest coo, or reversed male nest coo and also the corresponding LH levels. The peak LH concentrations sampled during $120 \mathrm{~min}$ of female nest coo stimulation were three times higher $(45.8 \pm 7.15 \mathrm{ng} / \mathrm{ml})$ than those sampled during stimulation with white noise $(15.51 \pm 4.18$ $\mathrm{ng} / \mathrm{ml})$ and those of control birds $(14.1 \pm 3.7 \mathrm{ng} / \mathrm{ml})$, which $\mathrm{did}$ not receive acoustic stimulation ( $p<0.01$; Fig. 10A1). A comparison of discharge rates in response to female nest coos versus 

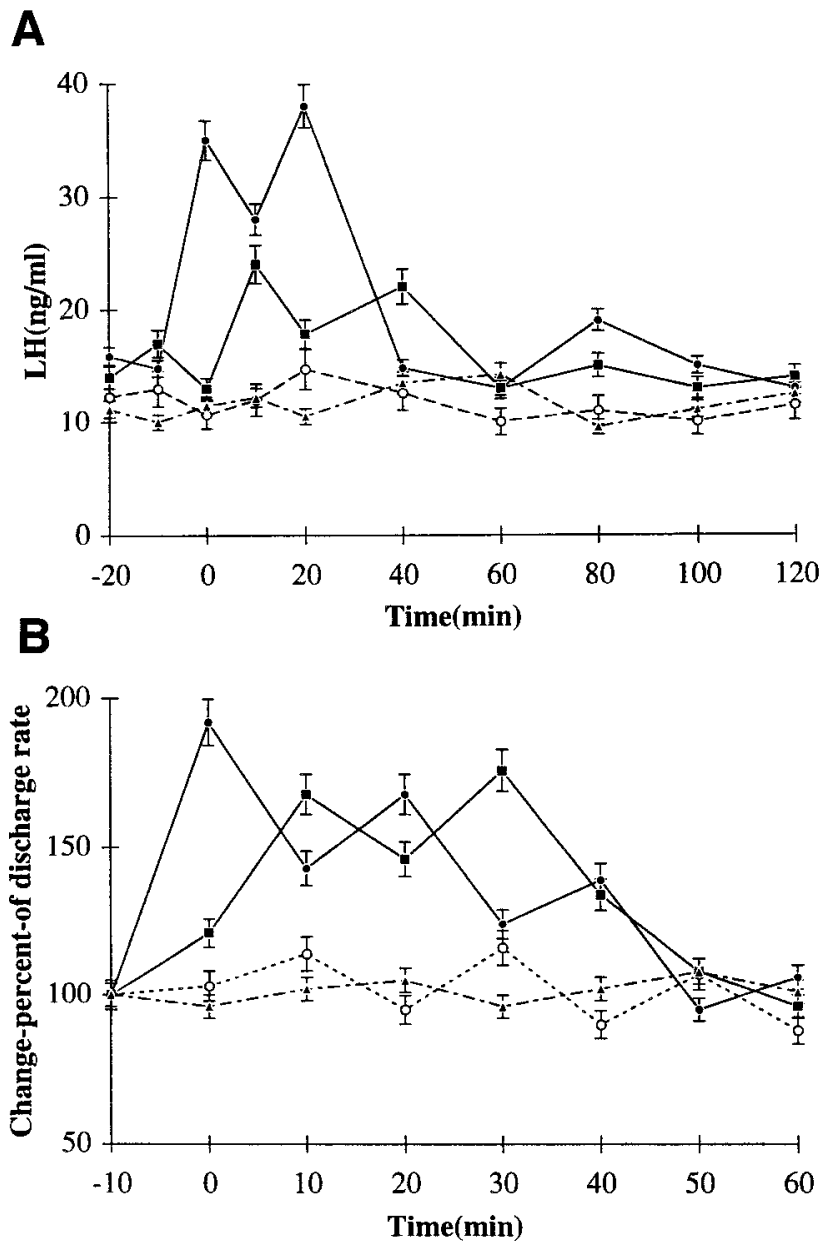

Figure 11. Effects of nest coo stimulation on neuronal response of anterior medial hypothalamus units and pituitary LH levels in female ring doves. There is a significant effect of different acoustic stimuli on $\mathrm{LH}$ levels $\left(F_{(3,36)}=6.91 ; p<.01\right)$ and on discharge rate $\left(F_{(3,31)}=4.67 ; p<\right.$ $0.01) . A, \mathrm{LH}$ levels were significantly higher for the female birds $(n=3)$ hearing female nest coo play backs (closed circles) than for those receiving no playback (closed triangles) at time points 0,10 , and $20 \mathrm{~min}$, in which $0=$ 40 min after the onset of female nest coo stimulation $(t=3.08 ; p<0.01)$. $\mathrm{LH}$ levels after hearing the male nest coo (closed squares) were also greater than LH levels in the control (closed triangle) $(t=2.81 ; p<0.05)$. Hearing the reversed nest coo (open circles: reversed female nest coo and reversed male nest coo combined), on the other hand, produced $\mathrm{LH}$ levels indistinguishable from those of the control $(t=0.09 ; p>0.05) . B$, Percentage changes of firing rate of units recorded during each sound stimulation. Closed circles, Female nest coo, responses of nine units; closed squares, male nest coo, responses of seven units; closed triangles, no playback, responses of five units; open circles, reversed male nest coo and reversed female nest coo combined, responses of seven units. Note that as in the preoptic area units shown in Figure 10, the percentage changes of neuronal response to the female nest coo and to the male nest coo are strikingly similar $(t=1.39 ; p>0.05)$.

white noise stimulation during a $0-40$ min time frame showed a significant difference ( $p<0.05$; Fig. 10A2). With reversed female nest coo stimulation, the peak LH level was indistinguishable from that of the control (Fig. 10A1, open circles). The male nest coo stimulation also resulted in an $\mathrm{LH}$ concentration (peak, $23.92 \pm 5.6 \mathrm{ng} / \mathrm{ml})$ higher than that of the control (15.54 \pm 4.92 $\mathrm{ng} / \mathrm{ml}$ ) (Fig. 10B1). The female nest coo induced significantly higher LH levels than the male nest coo (Fig. 10A1,B1).

We also asked whether there was a correlation between the magnitude of neuronal discharge and the level of LH output.
Using the change of firing rate of the control as $100 \%$, we calculated the percentage change of firing rate of POA neurons after 40 min stimulation of female nest coo versus male nest coo. We found 198 and $185 \%$ changes, respectively. The plots in Figure 10 are based on 114 POA units, 42 in response to female nest coos and 28 to reversed female nest coos, 16 to white noise, and 28 in the control group. Each point in the plot represents the sum total of discharges recorded in that period. The 42 units recorded in response to female nest coos during $60 \mathrm{~min}$ of stimulation can be broken down into 9 excitatory, 5 inhibitory, and 28 insignificant response units. The corresponding breakdown for the male nest coo plot in Figure $11 B 2$ is 5 excitatory, 4 inhibitory, and 32 unresponsive. The discharge rates for female and male nest coos were not statistically different (Fig. 10A2,B2).

In the $\mathrm{AMH}$, female nest coo stimulation evoked a $192 \%$ change in firing over the control; the corresponding LH levels (peak) were $38.92 \pm 7.4 \mathrm{ng} / \mathrm{ml}$, significantly different from the control (Fig. 11A). The male nest coo stimulation evoked an $184 \%$ firing rate change over the control (Fig. $11 \mathrm{~B}$ ); corresponding LH levels $(26.82 \pm 5.3 \mathrm{ng} / \mathrm{ml})$ were not as high as with female nest coo stimulation but were significantly different from those of the control (Fig. 11A). As in the POA, the female nest coo induced a significantly greater $\mathrm{LH}$ level than the male nest coo, although there was no statistical difference in discharge rate (Fig. $11 B$ ). Recording from female doves showed that when reversed female nest coos and reversed male nest coos were delivered, no changes in discharge rate or LH level were observed (Fig. 11A,B, open circles).

Figure 12 summarizes the results of all pituitary LH levels in response to the different acoustic stimuli. Although the male and female nest coos evoked a similar percentage change in the discharge rates of POA and $\mathrm{AMH}$ neurons (in the range of 170-200\%), the resulting LH concentrations in the pituitary were strikingly different. The female nest coo stimulation induced a magnitude of LH output three times (peak) greater than that of the male nest coo stimulation.

\section{DISCUSSION}

In discussing our findings, we need to consider the experimental conditions under which we obtained our data. First, the females we used for this study were 6 months old and had not been paired with males. At the time of the experiments, they had follicles of stage F5 and F4. Follicles of these stages measure $\sim 2-2.5 \mathrm{~mm}$; a preovulatory follicle measures $\sim 16-17 \mathrm{~mm}$ in this species. In other words, the LH output we observed was not part of the preovulatory LH surge. Second, follicles at these stages contain very low levels of estrogen, if any. Our LH data, therefore, reflect the GnRH response to acoustic stimulation with little or no estrogen priming. Third, blood samples were taken between 10 A.M. and 6 P.M., a period during which male and female pairs normally engage in vocal courtship exchange. Our LH data therefore mirrors what may actually transpire during the normal courtship cycle. Finally, assays of LH concentrations in the pituitary were independently performed in a laboratory that was not involved in the stimulation-recording experiments and therefore had no knowledge of the treatment groups.

We made the following predictions based on the evidence that led up to this study: (1) given the importance of the female nest coo in follicular growth of female doves (Cheng 1992, 1993), we predicted that the female nest coo would evoke the greatest LH response; and (2) given that the POA, the anterior medial hypothalamus, and the ventromedial nucleus receive axonal projec- 


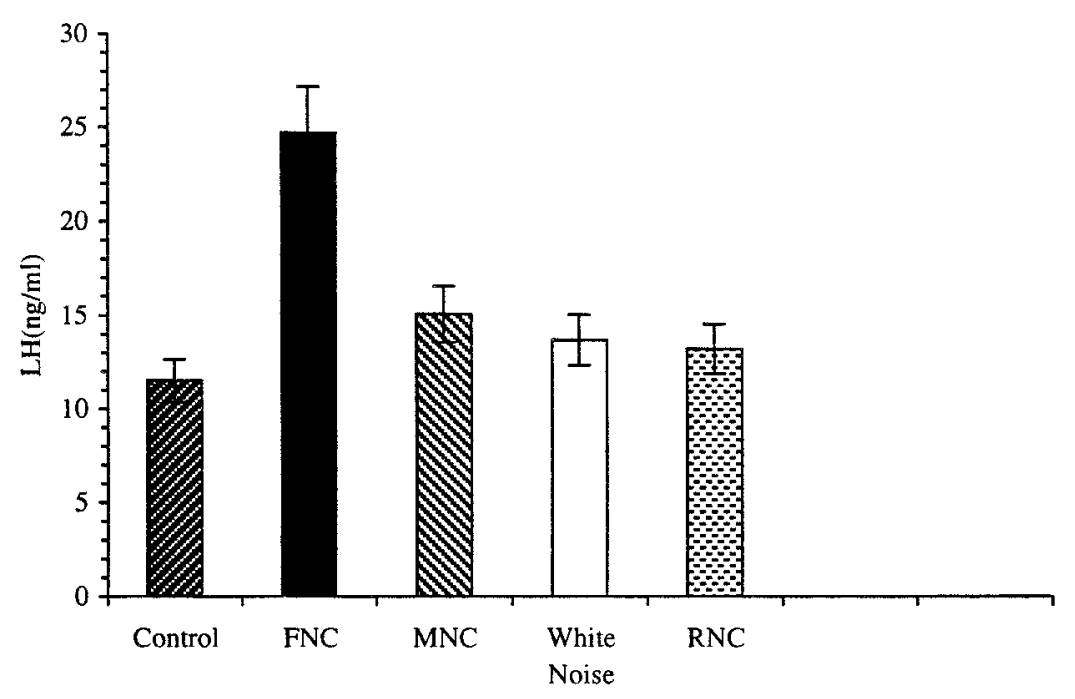

Figure 12. Histogram shows mean pituitary LH output radioimmunoassayed in birds exposed to different acoustic stimuli. The female nest coo group showed a significantly greater LH output than any of the other groups $\left(F_{(4,20)}=\right.$ $23.74 ; p<0.01)$. $F N C$, Female nest coo; $M N C$, male nest coo; $R N C$, reversed male nest coo and reversed female nest coo combined; Control, received no sound stimulation. tions from the nucleus ovoidalis (the auditory thalamic relay) and its shell region (Durand et al., 1992; Cheng and Peng, 1997) and from the midbrain vocal control nucleus (Cheng and Zuo, 1994), we predicted that units in these areas might respond differentially to the female and male nest coos.

The present analysis of $\mathrm{LH}$ data from females exposed to different coos and to white noise supports prediction 1 in that the female nest coo induced an LH level three times greater than that induced by the male nest coo. A response of $\mathrm{LH}$ release of this magnitude, presumably attributable to GnRH neuronal action, did not occur with any other acoustic stimulus. With regard to prediction 2, we found excitatory units in the POA, the $\mathrm{AMH}$, and the VMN that responded to the female nest coo but with a higher percentage of neurons in the POA and $\mathrm{AMH}$ responding selectively to the female nest coo. Indeed, the majority of VMN units were nonselective (Table 1). The discovery of a special kind of excitatory unit, i.e., the female-nest-coo-specific neuron, was entirely unexpected. These neurons were typically silent, responding only to the female nest coos and having a unique discharge pattern, i.e., two bursts of four to seven spikes separated by a silent period of 200-600 msec and a discharge duration of 1000-2000 msec (Fig. 5), approximately the same time frame as the two-note nest coo (female nest coo, $\sim 1100 \mathrm{msec}$; male nest coo, $\sim 1200 \mathrm{msec})$. The specificity of these neurons was further verified by their lack of response when the female nest coo was reversed. Interestingly, such neurons were very few in number. They constituted $\sim 2.6 \%(n=9)$ of the total units $(n=342)$ recorded in the POA and AMH combined.

Two forms of GnRH are widespread among avian species, chicken GnRH-I (cGnRH-I) and cGnRH-II. The general consensus is that the active form of GnRH for regulating anterior pituitary function is cGnRH-I (Sharp el al., 1990). The distribution pattern of neurons selectively sensitive to the female nest coo matches well with the overwhelming concentration of cGnRH-I neurons located in the POA (Kuenzel and Blahser, 1991; Silver et al., 1993; Cheng and Zuo, 1994). The distribution of cGnRH-I neurons extends to the AMH in turkeys (Millam et al., 1993) but is only sparsely present in ring doves (Cheng and Zuo, 1994). The close proximity of female-nest-coo-sensitive neurons to cGnRH-I neurons suggests that female-nest-coo-sensitive neurons may be cGnRH-I neurons or may synapse with cGnRH-I neurons.

The VMN of the ring dove, in which we found only inhibitory responses to the female nest coo, is completely void of any cGnRH-I neurons but dense with immunoreactive fibers (Silver et al., 1993; Cheng and Zuo, 1994). In the turkey, for which the distribution of both cGnRH-I and cGnRH-II are available, a high concentration of cGnRH-II in the VMN underscores the possibility that some of the inhibitory units we recorded in this region may be from or near cGnRH-II neurons and suggests that cGnRH-II neurons may play some role in reproduction.

Among the excitatory and inhibitory units responsive to female nest coo or male nest coo stimulation, some were clearly selective responses. Reversing the female nest coo or the male nest coo, for example, did not elicit bursting in previously responsive units. This selective responsiveness strongly suggests that units sensitive to the female nest coo, in particular the unique female-nest-coospecific units in the POA-AMH areas, can detect the temporal pattern of acoustic signals. The female-nest-coo-specific neurons exhibit a characteristic bursting response preceded and followed by silent or low discharge intervals. Similar patterned, selective responses were recently observed in the song-specific neurons of zebra finches (Lewicki, 1996) and in the electrosensory cells of electric fishes, which encode the temporal features of the stimulus waveform (Gabbiani et al., 1996).

The discharge pattern of female-nest-coo-specific neurons provides unmistakable cues that distinguish it from responses to the other acoustic stimuli (compare Figs. 4-6 with $2 B-D$ ). This may be a significant functional feature of the system in light of the fact that percentage discharge changes in the $\mathrm{POA}-\mathrm{AMH}$ region generated by the female and male nest coo stimulation were indistinguishable. The sum totals of excitatory and inhibitory discharges arriving at GnRH networks were similar whether the nest coos were female or male. However, the female nest coo stimulated a much higher level of $\mathrm{LH}$ release. We submit that one of the functions of female-nest-coo-specific neurons, with their unique response pattern, may be to send a "go" signal to GnRH or GnRH-related neurons. The concept of state space (Williams and Zipser, 1989) may be useful here to articulate how the female-nest-coo-responsive GnRH network might operate. The GnRH network may recognize the female-nest-coo-specific discharge as a prescribed instruction to move from a state of low response to a state of optimal LH release. In this model, femalenest-coo-specific discharges would have to appear only intermittently among a series of discharges from female-nest-coo- 
sensitive excitatory units. This may explain why there are so few female-nest-coo-specific neurons.

Recent studies on the synaptic inputs to GnRH neurons have yielded a consistent picture across different species of vertebrates. Catecholaminergic innervation of $\mathrm{GnRH}$ neurons and inhibitory inputs from opioid and GABA neurons have been reported in mammals as well as in birds (Muske, 1993; Silverman et al., 1994). We have identified the hypothalamic projection from the shell region of the nucleus ovoidalis as enkephalinergic (Durand et al., 1994). The projection originating from the vocal control nucleus is also enkephalinergic (Cheng and Zuo, 1994). These projections may exert inhibitory control of reproduction, because endogenous opioids on LH release are inhibitory (Cicero et al., 1979; Van Vugt et al., 1989).

Most neuroendocrine control of GnRH neurons appears to be inhibitory in nature. In addition to inhibitory effects of GABAergic and enkephalinergic systems on GnRH activity, transitory reduction of inhibitory action of 5-hydroxytryptamine and dopamine is involved in ovulation (Sharp et al., 1984). Inhibitory mechanisms also mediate ultrastructure changes in LHRH neurons in seasonal breeders (Parry and Goldsmith, 1993). Theoretical frameworks concerning the role of environmental and social factors in reproduction have not dealt with these neuroendocrine findings. Although the inhibitory nature of the effects of environmental stimuli on LH has been observed before (Yokoyama and Farner, 1976), the external cues have generally been thought to stimulate the hypothalamus-pituitary-ovarian (HPO) system (Ball, 1993; Wingfield et al., 1994). We cast the role of courtship in a different light. We propose that at least in birds, speciesspecific courtship behavior, such as the female nest coo, serves not so much to stimulate as to release inhibitory control of $\mathrm{GnRH}$ response (Cheng, 1993) - in other words, that the HPO system is normally kept in check until specialized courtship and/or environmental cues lift the inhibition. Selectively responsive inhibitory neurons may also facilitate GnRH output by suppressing their inhibitory control in response to the female nest coo. These inhibitory neurons may represent the network of inhibitory control of GnRH mentioned in the beginning of this paragraph. The inhibitory control of GnRH neurons is consistent with the observation in ring doves that ovarian follicles exhibit exponential growth once set in motion. Potential beneficiaries of the control of ovarian growth by disinhibition can be found in species of opportunistic breeders in which rapid onset of reproduction is best served by disinhibition. A beautiful example was recently documented in zebra finches, a species in which breeding is cued by water availability, in a study in which vasotocin administered by osmotic minipump inhibited breeding behavior within $2 \mathrm{~d}$ (Harding and Rowe, 1997).

The present finding on $\mathrm{LH}$ release is in general agreement with that of Everett et al. $(1976,1981)$ on LH release in rats on medial POA electrochemical stimulation. In the present study, the peak concentration of LH increase was observed after $\sim 40-60$ min of female nest coo stimulation (Fig. 11), a much faster rise than when electrochemical stimulation was used in rats, in which a latency of 90-120 min was recorded for peak LH (Colombo et al., 1974). Note also that the present study is not complicated by tissue loss, as was the case in experiments using electrochemical stimulation. With tissue loss, mechanisms of LH release through disinhibition were thought feasible (Dyball et al., 1976; Dyer et al., 1976). Based on the present findings, we suggest that both excitatory input and inhibitory input are likely to be involved.

\section{Concluding remarks}

We have identified female-nest-coo-sensitive excitatory and inhibitory neurons in the POA, AMH, and VMN. The femalenest-coo-specific neurons were found exclusively in the POA$\mathrm{AMH}$ areas and are characterized by a two-burst pattern with approximately the same temporal contours as the coo. After $<1$ hr of female nest coo stimulation, the pituitary $\mathrm{LH}$ concentration increased dramatically over the baseline concentration. The male nest coo also evoked an $\mathrm{LH}$ increment but with a much weaker response. Discharge changes recorded from units in the POAAMH further suggest that these units are involved in the elevation of LH output. These observations led us to conclude that GnRH networks in ring doves respond preferentially to the species-specific female nest coo.

\section{REFERENCES}

Bahr JM, Wang SC, Huang MY, Calvo FO (1983) Steroid concentrations in isolated theca and granulosa layers of preovulatory follicles during the ovulatory cycle of the domestic hen. Biol Reprod 29:326-334.

Ball GF (1993) The neural integration of environmental information by seasonally breeding birds. Am Zool 33:185-199.

Biederman-Thorson M (1970a) Auditory evoked responses in the cerebrum (field L) and ovoid nucleus of the ring dove. Brain Res 24:235-245

Biederman-Thorson M (1970b) Auditory responses of units in the ovoid nucleus and cerebrum (field L) of the ring dove. Brain Res 24:247-256.

Bronson FH (1989) Mammalian reproductive biology. Chicago: Chicago UP.

Capranica RR, Moffatt AJM (1983) Neurobehavioral correlates of sound communication in anurans. In: Advances in vertebrate neuroethology (Ewert RR, Capranica RR, Ingle D, eds), pp 701-730. New York: Plenum.

Cheng MF (1979) Progress and prospect in ring dove research. In: Advances in the study of behavior (Rosenblatt RA, Hinde RA, Beer CG, Busnel M-C, eds), pp 97-12. New York: Academic.

Cheng MF (1992) For whom does the female dove coo? A case for the role for the role of vocal self-stimulation. Anim Behav 43:1035-1044.

Cheng MF (1993) Vocal, auditory, and endocrine systems: three-way connectivites and implications. Poult Sci Rev 4:37-47.

Cheng MF, Peng JP (1997) Reciprocal talk between the auditory thalamus and the hypothalamus: an antidromic study. NeuroReport 8:653-658.

Cheng MF, Zuo M (1994) Proposed pathways for vocal self-stimulation: met-enkephalinergic projections linking the midbrain vocal nucleus, auditory-responsive thalamic regions, and neurosecretory hypothalamus. J Neurobiol 25:361-379.

Cheng MF, Desiderio C, Havens M, Johnson A (1988) Behavioral stimulation of ovarian growth. Horm Behav 22:388-402.

Cicero TJ, Schaikner B, Meyer ER (1979) Endogenous opioids participate in the regulation of the hypothalamic-pituitary-luteinizing hormone axis and testosterone's negative feedback control of luteinizing hormone. Endocrinology 104:1286-1290.

Colombo JA, Whitmoyer DI, Sawyer CH (1974) Local changes in multiple unit activity induced by electrochemical means in preoptic and hypothalamic areas in the female rat. Brain Res 71:1175-1183.

Conn PM (1994) The molecular mechanism of gonadotropin-releasing hormone action in the pituitary. In: The physiology of reproduction (Knobil E, Neill JD, eds), pp 1815-1832. New York: Raven.

Durand SE, Tepper JM, Cheng MF (1992) The shell region of the nucleus ovoidalis: a subdivision of the avian auditory thalamus. J Comp Neurol 323:495-518.

Dyball RE, Dyer RG, MacLeod NK, Wright RJ, Yates JO (1976) Effects of ferrous ions on secretion from incubated nerve terminals. J Endocrinol 72:73-82.

Dyer RG, Burnet F (1976) Effects of ferrous ions on preoptic area neurons and luteinizing hormone secretion in the rat. J Endocrinol 69:247-254.

Everett JW, Tyrey L (1981) Comparative increments of circulating luteinizing hormone in rats with increasing duration of electrical stimulation in medial preoptic or medial basal tuberal sites. Endocrinology 109:691-696. 
Everett JW, Quinn R, Tyrey L (1976) Comparative effectiveness of preoptic and tuberal stimulation for luteinizing hormone release and ovulation in two strains of rats. Endocrinology 98:1302-1308.

Gabbiani F, Metzner W, Wessel R, Koch C (1996) From stimulus encoding to feature extraction in weakly electric fish. Nature 384:564-567.

Harding CF, Rowe SA (1997) Vasotocin treatment inhibits courtship behavior in male zebra finches: concomitant androgen treatment inhibits this effect. Abstract presented at 27th Annual Meeting of the Society for Neuroscience, New Orleans, LA, October 25-30.

Harris GW (1955) Neural control of the pituitary gland. Plymouth, MA: Latime.

Huang SW, Tsai SC, Tung YF, Wang PS (1995) Role of progesterone in regulating the effect of estradiol on the secretion of thyrotropinreleasing hormone and dopamine into hypophyseal portal blood in ovariectomized rats. Neuroendocrinology 61:536-541.

Kroodsma DE (1976) Reproductive development in a female songbird: differential stimulation by quality of male song. Science 192:574-575.

Kuenzel WJ, Blahser S (1991) The distribution of gonadotropinreleasing hormone $(\mathrm{GnRH})$ neurons and fibers throughout the chick brain (Gallus domesticus). Cell Tissue Res 264:481-496.

Lehrman DS (1961) Gonadal hormones and parental behavior in birds and infrahuman mammals. In: Sex and internal secretions (Young WC, ed), pp 1268-1382. Baltimore: Williams \& Wilkens.

Lewicki MS (1966) Intracellular characterization of song-specific neurons in the zebra finch auditory forebrain. J Neurosci 16:5854-5863.

Margoliash D (1983) Acoustic parameters underlying the responses of song-specific neurons in the white-crowned sparrow. J Neurosci 3:1039-1057.

Marshall FHA (1942) Exteroceptive factors in sexual periodicity. Biol Rev 17:68-90.

Millam JR, Faris PL, Youngren OM, El Halawani ME, Hartman BK (1993) Immunohistochemical localization of chicken gonadotropinreleasing hormones I and II (cGnRHI and II) in turkey hen brain. J Comp Neurol 333:62-82.

Muske LE (1993) Evolution of gonadotropin-releasing hormone (GnRH) neuronal systems. Brain Behav Evol 42:215-230.

Parry DM, Goldsmith AR (1993) Ultrastructural evidence for changes in synaptic input to the hypothalamic luteinizing hormone-releasing hormone neurons in photosensitive and photorefractory starlings. J Neuroendocrinol 5:387-395.

Peng JP, Qiao HL, Yang CR (1996) A high correlation between the electric activity of neurons in preoptic/anterior hypothalamic area and
LH release from the hypophysis. In 6th International symposium on avian endocrinology (Harvey S, ed). Poult Avian Biol Rev (abstr) 6:206.

Scanes CG, Follett BK (1972) Fractionation and assay of chicken pituitary hormones. Br Poult Sci 13:603-610.

Sharp PJ, Macnamee MC, Talbot RT, Sterling RJ, Hall TR (1984) Aspects of the neuroendocrine control of ovulation and broodiness in the domestic hen. J Exp Zool 232:475-483.

Sharp PJ, Dunn IC, Talbot RT (1987) Sex differences in the LH response to chicken LHRH-I and -II in the domestic fowl. J Endocrinol 115:323-331.

Sharp PJ, Duncan IC, Main GM, Sterling RJ, Talbot RT (1990) Gonadotropin-releasing hormones: distribution and function. In: Endocrinology of birds (Wada M, Ishii S, Scanes GC, eds), pp 31-42. Berlin: Springer.

Silver R, Ramos C, Machca H, Silverin B (1992) Immunocytochemical distribution of GnRH in the brain of adult and posthatching great tit (Parus major) and ring dove (Streptopelia roseogrisea). Ornis Scand 23:222-232.

Silverman A-J, Livne I, Witkin JW (1994) The gonadotropin-releasing hormone (GnRH), neuronal systems: immunocytochemistry and in situ hybridization. In: The physiology of reproduction (Knobil E, Neill JD, eds), pp 1683-1709. New York: Raven.

Thind KK, Goldsmith PC (1988) Infundibular gonadotropin-releasing hormone neurons are inhibited by direct opioid and autoregulatory synapses in juvenile monkeys. Neuroendocrinology 47:203-216.

Van Vugt DA, Lam NY, Ferin M (1984) Reduced frequency of pulsatile luteinizing hormone secretion in the luted phase of the rhesus monkey: involvement of endogenous opiates. Endocrinology 115:1095-1102.

Wilczynski W, Allison JD, Marler CA (1993) Sensory pathways linking social and environmental cues to endocrine control regions of amphibian forebrains. Brain Behav Evol 42:252-264.

Williams RJ, Zipser D (1989) Experimental analysis of the real time recurrent learning algorithm. Connect Sci 1:87-111.

Wingfield JC, Whaling CS, Marler P (1994) Communication in vertebrate aggression and reproduction: the role of hormones. In: The physiology of reproduction (Knobil E, Neill JD, eds), pp 303-342. New York: Raven.

Yokoyama K, Farner DS (1976) Photoperiodic responses in bilaterally enucleated female white-crowned sparrows, Zonotrichia Leucophrys gambelii. Gen Comp Endocrinol 30:528-533.

Yu AC, Margoliash D (1996) Temporal hierarchical control of singing in birds. Science 173:1871-1875. 